\title{
Sensor-To-Sensor Data Association and Preliminary Estimation \\ of Coordinates of Air Targets in Multisensory Radar in Multitarget Conditions
}

\author{
Vladislav V. Kiryushkin*a, \\ Alexey V. Korovina and Alexander V. Zhuravlev \\ ${ }^{a}$ Military Education and Research Centre of Military-Air Forces \\ "Military-Air Academy \\ named after Professor N.E. Zhukovsky and Yu.A. Gagarin” \\ 54a Starykh Bolshevikov Str., Voronezh, 394064, Russia \\ bJoint-stock company "Scientific-Promotional enterprise "PROTEK" \\ 6 Bazovaya Str., Voronezh, 394028, Russia \\ Received 08.11.2018, received in revised form 07.03.2019, accepted 24.03.2019 \\ The new method of data association and preliminary estimation of coordinates of air targets in \\ multisensory radar is offered. The method operates in the multitarget conditions when trajectories \\ of the targets are not tracked yet and all targets are observed simultaneously. The method is based \\ on virtual survey of space, uses only total-range measurements and does not demand measurement
} of additional parameters.

Keywords: multisensory radar, multitarget conditions, data association, virtual survey of space.

Citation: Kiryushkin V.V., Korovin A.V., Zhuravlev A.V. Sensor-to-sensor data association and preliminary estimation of coordinates of air targets in multisensory radar in multitarget conditions, J. Sib. Fed. Univ. Eng. technol., 2019, 12(6), 708-718. DOI: $10.17516 / 1999-494 X-0170$.

(c) Siberian Federal University. All rights reserved

This work is licensed under a Creative Commons Attribution-NonCommercial 4.0 International License (CC BY-NC 4.0).

* Corresponding author E-mail address: kiryushkin.vlad@mail.ru 


\title{
Межпозиционное отождествление
}

\author{
результатов измерений и определение \\ координат воздушных целей
}

\section{в многопозиционной радиолокационной системе \\ в условиях многоцелевой обстановки}

\author{
В.В. Кирюшкин ${ }^{a}$ А.В. Коровин ${ }^{\text {a }}$ А.В. Журавлев ${ }^{\sigma}$ \\ ${ }^{a}$ Военный учебно-научный центр Военно-воздушных сил \\ «Военно-воздушная академия \\ имени профессора Н.Е. Жуковского и Ю.А. Гагарина» \\ Россия, 394064, Воронеж, ул. Старых Большевиков, 54 а \\ ${ }^{6}$ Акиионерное общество \\ «Научно-внедренческое предприятие «ПРОТЕК» \\ Россия, 394028, Воронеж, ул. Базовая, 6
}

\begin{abstract}
Предложен новый способ межпозиционного отождествления измерений и предварительного определения координат воздушных иелей в суммарно-дальномерной многопозиционной радиолокаиионной системе в условиях многочелевой обстановки, когда завязка траекторий целей еще не произошла и все цели наблюдаются одновременно. Способ основан на виртуальном обзоре пространства, использует только суммарно-дальномерные измерения и не требует измерения дополнительных параметров.
\end{abstract}

Ключевые слова: многопозиционная радиолокачионная система, многоцелевая обстановка, межпозиционное отождествление, виртуальный обзор пространства.

\section{Введение}

При одновременном наблюдении нескольких целей в многопозиционной радиолокационной системе (МПРЛС) возникает актуальная практическая задача определения принадлежности полученных в разных позициях измерений тем или иным целям. Перепутывание данных в процессе их объединения может привести к грубым ошибкам определения координат целей [1]. Решение этой задачи осуществляется в ходе операции межпозиционного отождествления данных [1-8].

Обычно под отождествлением результатов измерений понимают процесс принятия решения о соответствии полученных результатов измерений той или иной уже наблюдаемой цели. При этом процесс отождествления измерений включает в себя два этапа: 1) сопоставление результатов измерений со всеми фильтруемыми траекториями; 2) выбор одной траектории, наиболее достоверно соответствующей результатам измерений, или завязка новой траектории [1, 8]. Однако такой способ применим только в условиях фильтрации уже завязанных траекторий одной или нескольких целей.

В настоящей работе предложен способ межпозиционного отождествления измерений и определения координат целей в МПРЛС в условиях многоцелевой обстановки, когда завязка траекторий целей еще не произошла и все цели наблюдаются одновременно.

$$
-709-
$$




\section{Постановка задачи}

Рассмотрим МПРЛС, состоящую из $M$ синхронизированных специализированных передатчиков $T R_{i}$, расположенных в точках с координатами $\left(x_{i}^{T R}, y_{i}^{T R}, z_{i}^{T R}\right)$, где $i=1,2, \ldots, M$, и $N$ приемников $R S_{j}$, расположенных в точках с координатами $\left(x_{j}^{R S}, y_{j}^{R S}, z_{j}^{R S}\right)$, где $j=1,2, \ldots, N$, в зоне действия которой одновременно находятся $K$ целей $T_{m}$, где $m=1,2 \ldots K$. Суммарные дальномерные измерения осуществляются по $(M \mathrm{x} N)$ бистатическим звеньям «TR $-R S »$ (рис. 1).

В каждом бистатическом звене наблюдаются отклики от $K$ целей, траекторные параметры которых априорно неизвестны. Суммарные измерения дальности по сигналу от $i$-го передатчика $T R_{i}$, рассеянному $m$-й воздушной целью $T_{m}$ и принятому в $j$-м приемнике $R S_{j}$, будут иметь вид

$$
r_{j, m}^{\mathrm{i}}=r_{i, m}^{T R}+r_{j, m}^{R S}+\varepsilon,
$$

где $\quad r_{i, m}^{T R}=\left[\left(x_{i}^{T R}-x_{m}\right)^{2}+\left(y_{i}^{T R}-y_{m}\right)^{2}+\left(z_{i}^{T R}-z_{m}\right)^{2}\right]^{1 / 2} \quad-\quad$ дальность пути $\quad$ пTR $-T_{m} » ;$ $r_{j, m}^{R S}=\left[\left(x_{j}^{R S}-x_{m}\right)^{2}+\left(y_{j}^{R S}-y_{m}\right)^{2}+\left(z_{j}^{R S}-z_{m}\right)^{2}\right]^{1 / 2}$ - дальность пути « $T_{m}-\mathrm{RS}_{j} »,\left(x_{m}, y_{m}, z_{m}\right)-$ координаты $m$-й воздушной цели $T_{m} ; \varepsilon-$ случайная погрешность измерения дальности.

В ходе процесса первичного межпозиционного отождествления результатов измерений в МПРЛС необходимо все суммарные измерения дальности по сигналу от передатчика $T R_{i}$, pacсеянному $m$-й воздушной целью $T_{m}$ и принятому в приемнике $R S_{j} r_{j, m}^{\mathrm{i}}$, поставить в соответствие нужной цели $T_{m}$, сформировать $K$ векторов измерений $\mathbf{R}_{m}$, где $m=1 \ldots K$, грубо определить координаты всех $K$ наблюдаемых целей и подготовить исходные данные для завязки траекторий.

\section{Способ межпозиционного отождествления измерений}

\section{и определения координат целей в МПРЛС в условиях многоцелевой обстановки}

Наиболее широко известен способ первичного межпозиционного отождествления результатов измерений в МПРЛС, основанный на прямом переборе всех возможных гипотез отождествления [1]. Однако в этом случае потребуется проверить $(K !)^{(N \cdot M-1)}$ гипотез, для каждой из которых вычислить координаты $K$ целей с использованием известных методов (например,

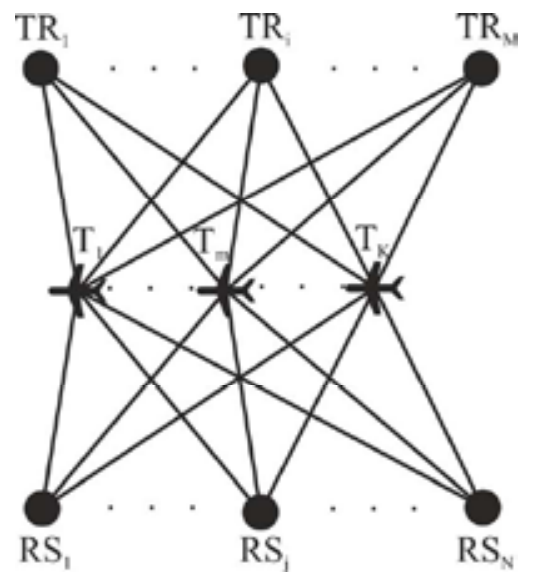

Рис. 1. Конфигурация МПРЛС в горизонтальной плоскости в условиях многоцелевой обстановки

Fig. 1. Configuration of multisensory radar in a horizontal plane in multitarget conditions 
метода наименьших квадратов). При этом количество гипотез, а следовательно, и потребные вычислительные мощности быстро растут с увеличением количества наблюдаемых целей [1].

Для сокращения количества ложных гипотез применяется предварительное стробирование по координатам и параметрам, для чего необходимы дополнительные избыточные измерения, например, в дальномерных измерительных системах необходимо измерить и азимут цели [1].

Предложенный способ первичного межпозиционного отождествления обеспечивает сокращение количества гипотез отождествления и не требует при этом дополнительных избыточных измерений. Способ основан на виртуальном обзоре пространства [3] по координатам $x$ и $y$ с шагом, соответствующим разрешающей способности МПРЛС по дальности. При этом на каждом шаге сканирования в пределах зоны действия МПРЛС тем или иным способом задается предполагаемое (гипотетическое) местоположение цели на плоскости $T^{H}(x, y)$. Предполагаемую высоту цели выбирают фиксированной, соответствующей середине зоны действия МПРЛС по высоте $\bar{z}$. Для предполагаемого местоположения цели рассчитывают предполагаемые суммарные дальности в каждом бистатическом звене «TR ${ }_{i}$ $\left.T^{H}-R S_{j}\right\rangle$ :

$$
\begin{aligned}
& r r_{j}^{\mathrm{i}}(x, y)=\left[\left(x_{i}^{T R}-x\right)^{2}+\left(y_{i}^{T R}-y\right)^{2}+\left(z_{i}^{T R}-\bar{z}\right)^{2}\right]^{1 / 2}+ \\
& +\left[\left(x_{j}^{R S}-x\right)^{2}+\left(y_{j}^{R S}-y\right)^{2}+\left(z_{j}^{R S}-\bar{z}\right)^{2}\right]^{1 / 2} .
\end{aligned}
$$

Для соответствующего бистатического звена из $K$ действительных измерений $r_{j, m}^{\mathrm{i}}$ выбирают измерение, наилучшее по критерию минимизации модуля невязки между предполагаемым и действительным измерением:

$$
\hat{m}=\arg \min \left(\left|r r_{j}^{\mathrm{i}}(x, y)-r_{j, m}^{\mathrm{i}}\right|\right) .
$$

Для всей МПРЛС, т.е. для всей совокупности бистатических звеньев, для предполагаемого местоположения цели определяется суммарная невязка:

$$
\Delta r_{\Sigma}(x, y)=\sum_{i=1}^{M} \sum_{j=1}^{N}\left|r r_{j}^{\mathrm{i}}(x, y)-r_{j, \hat{m}}^{\mathrm{i}}\right| \text {. }
$$

Проверка наличия цели в предполагаемом ее местоположении $T^{H}(x, y)$ осуществляется после виртуального сканирования всей зоны действия МПРЛС и только в точках минимума суммарной невязки. Для обострения минимумов и лучшего их выявления целесообразно использовать логарифм от значения суммарной невязки. В этом случае проверка наличия цели будет осуществляться в точках $(\hat{x}, \hat{y})$, определяемых в соответствии с выражением

$$
\frac{d^{2} \lg \left(\Delta r_{\Sigma}(x, y)\right)}{d x d y}=0 .
$$

При этом минимумы суммарной невязки будут формироваться для каждой из $K$ наблюдаемых целей, а также для ложных целей.

Для исключения ложных целей значение функции в проверяемых точках минимума $\lg \left(\Delta r_{\Sigma}(\hat{x}, \hat{y})\right)$ сравнивают с порогом $h$, величина которого определяется с учетом погрешности 
$\Delta_{\varepsilon}$ дальномерных измерений, погрешности $\Delta_{x, y}$, обусловленной конечным значением шага виртуального обзора пространства по координатам $x$ и $y$, а также погрешности $\Delta_{z}$, обусловленной неточностью определения высоты целей, для каждого бистатического звена:

$$
h=\lg \left(N \cdot M \cdot\left(\Delta_{\varepsilon}+\Delta_{x, y}+\Delta_{z}\right)\right) .
$$

Погрешность дальномерных измерений определяется как максимальная ошибка измерений

$$
\Delta_{\varepsilon}=3 \sigma_{\varepsilon},
$$

где $\sigma_{\varepsilon}$ - среднеквадратическое отклонение (СКО) случайной погрешности измерения дальности $\varepsilon$, распределенной по нормальному закону с нулевым математическим ожиданием.

Погрешность, обусловленная конечным значением шага виртуального обзора пространства, определяется как наибольшее удаление цели от проверяемой точки с учетом, что проверяемая точка соответствует центру ячейки обзора пространства, а цель находится в ее узле:

$$
\Delta_{x, y}=\sqrt{\left(0.5 \Delta_{x}\right)^{2}+\left(0.5 \Delta_{y}\right)^{2}},
$$

где $\Delta_{x}$ и $\Delta_{y}$ - величина шага виртуального обзора пространства по координатам $x$ и $y$ соответственно.

Погрешность, обусловленная неточностью определения высоты целей, может быть определена как максимальная разность хода лучей «TR $-T-R S »$ для случаев, когда цель находится на средней высоте $\bar{z}$ и у поверхности земли:

$$
\Delta_{z}=2\left(\sqrt{(0.5 L)^{2}+\bar{z}^{2}}-0.5 L\right)
$$

где $L$ - база МПРЛС (расстояние между линией передатчиков и приемников).

Принятие окончательного решения о наличии $(\theta=1)$ или отсутствии $(\theta=0)$ цели в проверяемой точке пространства осуществляется в соответствии с правилом

$$
\lg \left(\Delta r_{\Sigma}(\hat{x}, \hat{y})\right) \frac{>h \Rightarrow \theta=0}{\leq h \Rightarrow \theta=1}
$$

Координаты проверяемых точек $(\hat{x}, \hat{y})$, для которых выполняется правило (10), являются грубой оценкой координат наблюдаемых целей $\left(\hat{x}_{m}, \hat{y}_{m}\right)$.

Вектор измерений $\mathbf{R}_{m}$ для каждой $m$-й воздушной цели $T_{m}$ будет сгруппирован из измерений $r_{j, \hat{m}}^{\mathrm{i}}$, обеспечивающих минимум суммарной невязки $\lg \left(\Delta r_{\Sigma}\right)$ в соответствующей точке $\left(\hat{x}_{m}, \hat{y}_{m}\right)$.

Точное определение координат каждой $m$-й воздушной цели $T_{m}$ осуществляют на основании сформированных векторов измерений $\mathbf{R}_{m}$ одним из известных методов, например методом наименьших квадратов [9].

Таким образом, эффективность предложенного способа в сравнении с прямым перебором всех возможных гипотез определяют сокращением количества проверяемых гипотез с $(K !)^{(N \cdot M-1)}$ до одной. 


\section{Методика исследования эффективности способа межпозиционного отождествления измерений и определения координат целей в МПРЛС}

Исследование эффективности способа межпозиционного отождествления измерений и определения координат целей в МПРЛС было проведено методом имитационного моделирования. Структурная схема имитационной модели приведена на рис. 2.

В блоке 1 задается конфигурация МПРЛС путем определения матрицы $\mathbf{X}^{T R}$ координат передающих позиций $\left(x_{i}^{T R}, y_{i}^{T R}, z_{i}^{T R}\right)$, где $i=1,2, \ldots, M$, и матрицы $\mathbf{X}^{R S}$ координат приемных позиций $\left(x_{j}^{R S}, y_{j}^{R S}, z_{j}^{R S}\right)$, где $j=1,2, \ldots, N$. В блоке 3 задается матрица $\mathbf{X}^{T}$ координат воздушных целей $\left(x_{m}, y_{m}, z_{m}\right)$, где $m=1,2, \ldots, K$. В блоке 2 на основании заданной конфигурации МПРЛС и заданных координат одновременно наблюдаемых воздушных целей в соответствии с (1) для каждого бистатического звена для каждой цели рассчитывают суммарные измерения дальности $r_{j, m}^{\mathrm{i}}$ по сигналу от $i$-го передатчика $T R_{i}$, рассеянному $m$-й воздушной целью $T_{m}$ и принятому в $j$-м приемнике $R S_{j}$ с учетом заданного СКО погрешности дальномерных измерений $\sigma_{\varepsilon}$.

В блоке 4 в пределах зоны действия МПРЛС с заданным шагом по координате $x$ и координате $y$ на средней высоте $\bar{z}$ задают предполагаемое местоположение цели $T^{H}(x, y)$. В блоке 5 для предполагаемого местоположения цели в соответствии с (2) рассчитывают предполагаемые суммарные дальности $r r_{j}^{\mathrm{i}}(x, y)$ в каждом бистатическом звене «TR ${ }_{i}-T^{H}-R S_{j} »$. Вычисление модуля невязки между предполагаемым и действительными измерениями в каждом бистатическом звене осуществляется в блоке 6 . При этом из $K$ действительных измерений $r_{j, m}^{\mathrm{i}}$ выбирают измерение $r_{j, \hat{m}}^{\mathrm{i}}$, наилучшее по критерию (3).

В блоке 9 в соответствии с (4) определяют логарифм суммарной невязки $\lg \left(\Delta r_{\Sigma}(x, y)\right)$ и в соответствии с (5) определяют точки $\left(\hat{x}_{m}, \hat{y}_{m}\right)$, в которых его значение будет минимальным. Полученные минимальные значения $\lg \left(\Delta r_{\Sigma}(\hat{x}, \hat{y})\right)$ в блоке 8 сравнивают с порогом $h$, значение которого рассчитывают в блоке 7 на основании известных $\Delta_{x}, \Delta_{y}, \bar{z}, \sigma_{\varepsilon}$. Если значение $\lg \left(\Delta r_{\Sigma}(\hat{x}, \hat{y})\right)$ удовлетворяет условию (10), то по сигналу $\theta=1$ в блоке 11 координаты соответствующей точки $(\hat{x}, \hat{y})$, поступающие из блока 4 , выбирают в качестве грубой оценки ко-

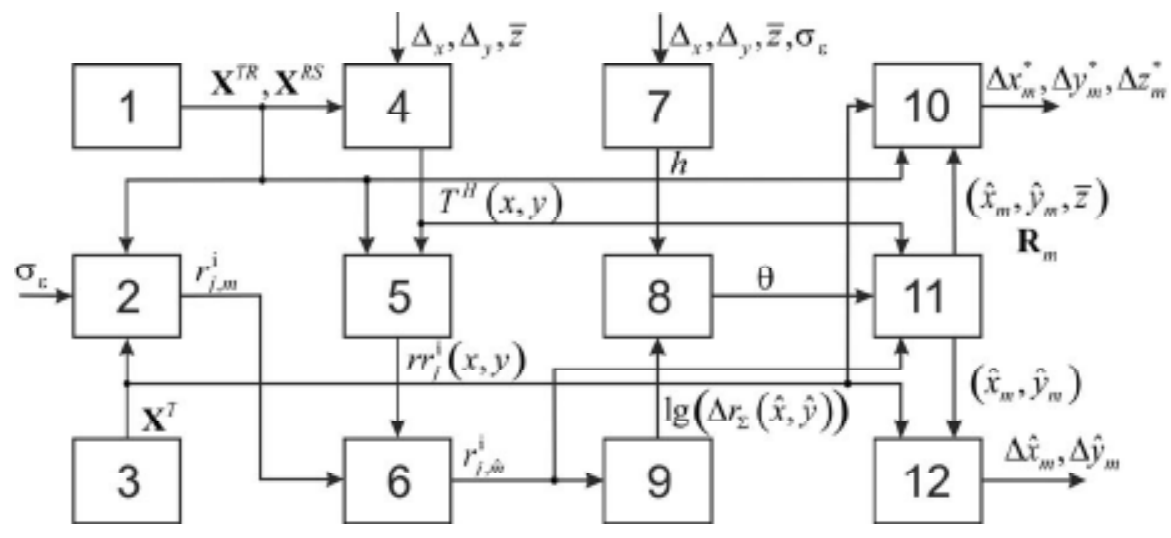

Рис. 2. Структурная схема имитационной модели

Fig. 2. The block diagramme of imitating model 
ординат наблюдаемой $m$-й воздушной цели $\left(\hat{x}_{m}, \hat{y}_{m}\right)$. При этом вектор измерений $\mathbf{R}_{m}$ для $m$-й воздушной цели $T_{m}$ будет сгруппирован из измерений $r_{j, \hat{m}}^{\mathrm{i}}$, поступающих из блока 6.

Непосредственная оценка эффективности предложенного способа в плане определения координат целей заключается в сравнении в блоке 12 полученных оценок координат $\left(\hat{x}_{m}, \hat{y}_{m}\right)$ целей на плоскости с заданными координатами воздушных целей $\left(x_{m}, y_{m}\right)$, поступающими из блока 3 , и формировании сигналов рассогласования $\Delta \hat{x}_{m}, \Delta \hat{y}_{m}$.

Для оценки эффективности межпозиционного отождествления измерений предложенным способом сформированные векторы измерений $\mathbf{R}_{m}$ используют в блоке 10 в качестве исходных измерений для более точного метода определения координат (метода наименьших квадратов), при этом полученные грубые оценки координат целей $\left(\hat{x}_{m}, \hat{y}_{m}, \bar{z}\right)$ применяют в качестве начального приближения для получения точного решения координатной задачи $\left(x_{m}^{*}, y_{m}^{*}, z_{m}^{*}\right)$ этим итерационным методом. Степень отклонения $\Delta x_{m}^{*}, \Delta y_{m}^{*}, \Delta z_{m}^{*}$ полученной точной оценки $\left(x_{m}^{*}, y_{m}^{*}, z_{m}^{*}\right)$ от заданных координат $\left(x_{m}, y_{m}, z_{m}\right)$ воздушных целей используется в качестве критерия эффективности межпозиционного отождествления измерений в МПРЛС.

\section{Результаты численного эксперимента}

В соответствии с описанной методикой численный эксперимент был проведен для МПРЛС, состоящей из $M=3$ передатчиков и $N=3$ приемников, разнесенных на ширину базы $L=40000 \mathrm{M}$. В зоне действия МПРЛС одновременно находятся $K=7$ целей. Координаты воздушных целей в локальной системе координат, за начало которой принят центр МПРЛС, а также координаты приемных и передающих позиций МПРЛС приведены в табл. 1.

Геометрия численного эксперимента на плоскости показана на рис. 3, где передающие позиции обозначены квадратами, приемные позиции - ромбами, а кружки демонстрируют истинное местоположение воздушных целей.

При численном моделировании использовали следующие значения параметров:

- СКО случайной погрешности измерения дальности $\sigma_{\varepsilon}=10$ м;

- величина шага обзора по координатам $x$ и $y \Delta_{x}=\Delta_{y}=100$ м;

- $\quad$ середина зоны действия МПРЛС по высоте соответствует $\bar{z}=1000$ м.

На рис. 4 показано полученное в ходе моделирования поле логарифма суммарной невязки $\lg \left(\Delta r_{\Sigma}(x, y)\right)$. Видно, что на графике наблюдаются восемь основных минимумов (обозначены цифрами 1 - 8), семь из которых соответствуют семи истинным целям, а один - ложной цели.

Полученные точки $\left(\hat{x}_{m}, \hat{y}_{m}\right)$, в которых значение логарифма суммарной невязки $\lg \left(\Delta r_{\Sigma}(x, y)\right)$ минимально, изображены на рис. 3 шестиконечными звездами. Видно, что пер-

Таблица 1. Координаты целей и позиций МПРЛС

Table 1. Coordinates of the targets and of the positions of multisensory radar

\begin{tabular}{|c|c|c|c|c|c|c|c|c|c|c|c|c|c|}
\hline Коорд. & $\mathrm{TR}_{1}$ & $\mathrm{TR}_{2}$ & $\mathrm{TR}_{3}$ & $\mathrm{RS}_{1}$ & $\mathrm{RS}_{2}$ & $\mathrm{RS}_{3}$ & $\mathrm{~T}_{1}$ & $\mathrm{~T}_{2}$ & $\mathrm{~T}_{3}$ & $\mathrm{~T}_{4}$ & $\mathrm{~T}_{5}$ & $\mathrm{~T}_{6}$ & $\mathrm{~T}_{7}$ \\
\hline $\mathrm{x}, \mathrm{M}$ & 0 & -20000 & 20000 & 0 & -20000 & 15000 & 6050 & 8030 & 17090 & -10040 & -15070 & -5010 & 10120 \\
$\mathrm{y}, \mathrm{M}$ & 20000 & 17000 & 25000 & -20000 & -20000 & -22000 & 10070 & -1010 & 5080 & 7150 & 8090 & -5070 & -10060 \\
$\mathrm{z}, \mathrm{M}$ & 0 & 0 & 0 & 0 & 0 & 0 & 500 & 400 & 800 & 1400 & 1100 & 600 & 300 \\
\hline
\end{tabular}




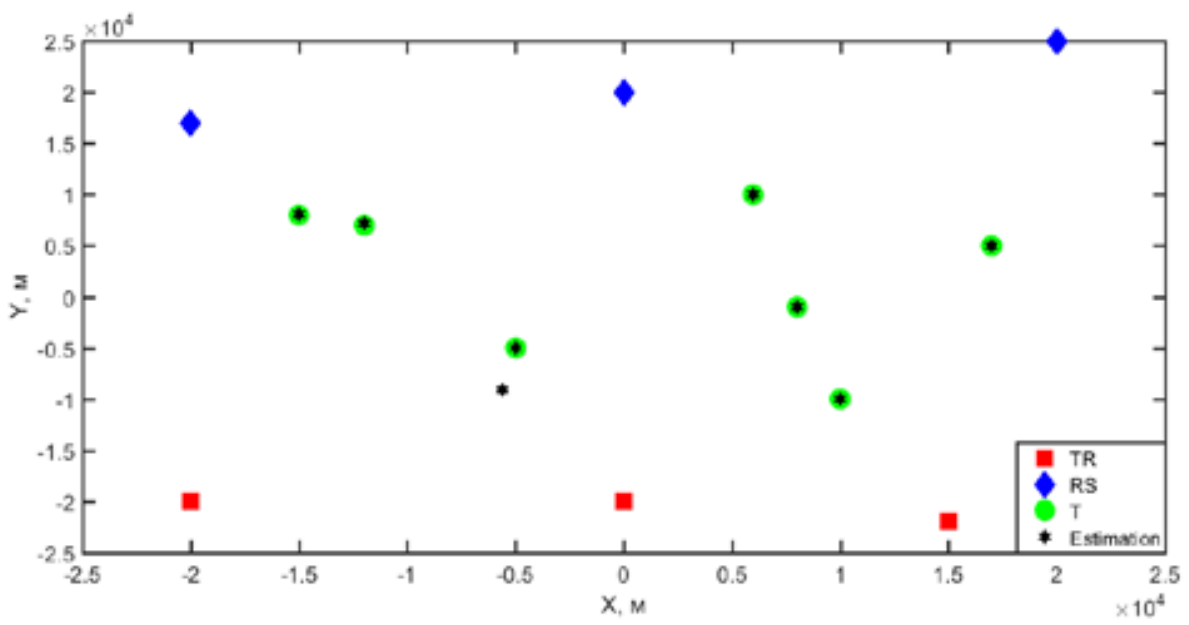

Рис. 3. Геометрия численного эксперимента

Fig. 3. Geometry of numerical experiment

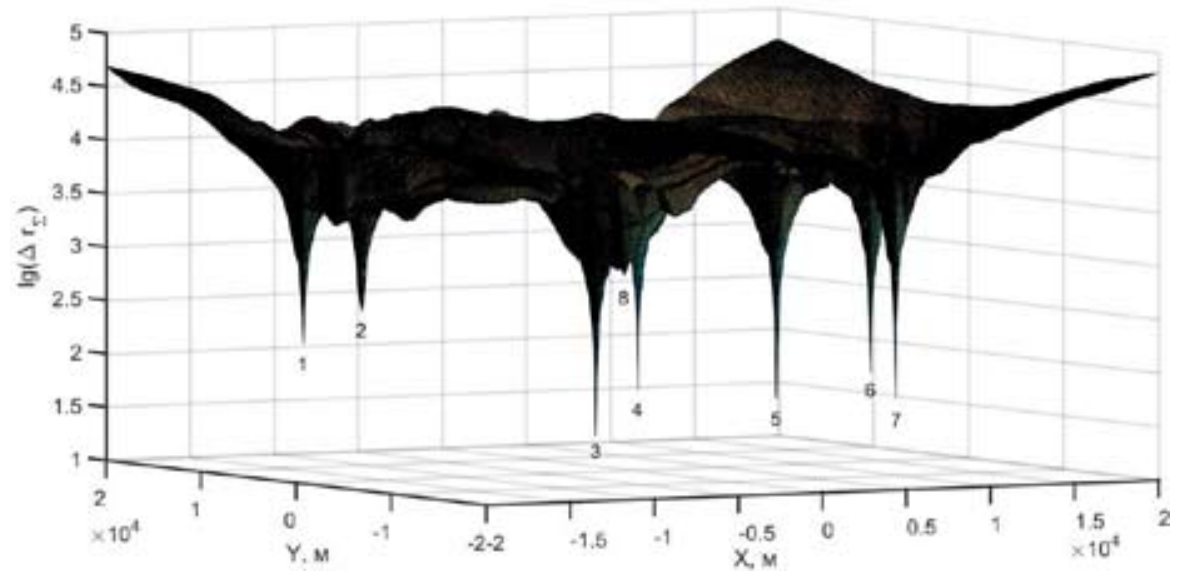

Рис. 4. Пространственное распределение логарифма суммарной невязки

Fig. 4. Spatial allocation of logarithm of total discrepancy of measurements

вые семь оценок $\left(\hat{x}_{m}, \hat{y}_{m}\right)$ практически полностью совпали с местоположением истинных целей, а восьмая оценка не совпадает ни с одной целью.

Для исключения ложных целей значение функции в проверяемых точках минимума $\lg \left(\Delta r_{\Sigma}(\hat{x}, \hat{y})\right)$ в соответствии с (10) сравнивалось с порогом $h$, величина которого была рассчитана в соответствии с (6) - (9) и составила значение $h=2.96$.

Соотношение между значениями $\lg \left(\Delta r_{\Sigma}(\hat{x}, \hat{y})\right)$ для всех восьми проверяемых точек минимума суммарной невязки и значением порога изображено на рис. 5 , где по горизонтальной оси отложен номер проверяемой точки по мере возрастания суммарной невязки, а по вертикальной оси отложено само значение невязки. Проверяемые значения $\lg \left(\Delta r_{\Sigma}(\hat{x}, \hat{y})\right)$ показаны жирными крестиками, а пороговое значение $h$ - горизонтальной линией. Видно, что первые семь точек минимума суммарной невязки удовлетворяют критерию (10) и соответствующие координаты 


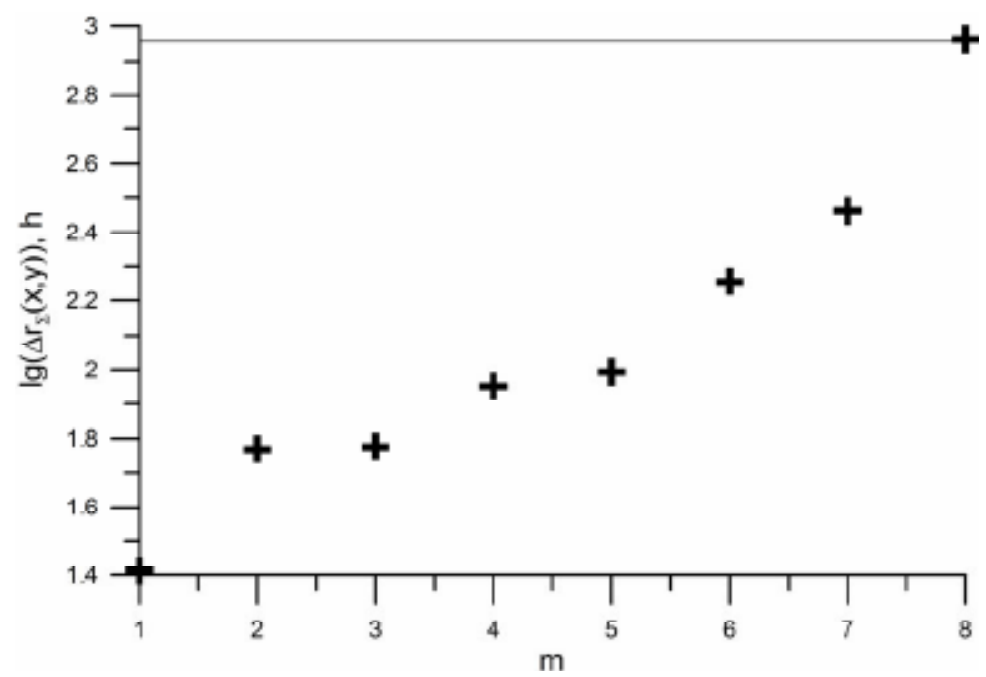

Рис. 5. Соотношение между $\lg \left(\Delta r_{\Sigma}(\hat{x}, \hat{y})\right)$ для восьми точек минимума суммарной невязки и значением порога $h$

Fig. 5. Ratio between $\lg \left(\Delta r_{\Sigma}(\hat{x}, \hat{y})\right)$ for eight points of a minimum of total discrepancy of measurements and value of threshold $h$

этих точек $(\hat{x}, \hat{y})$ могут быть выбраны в качестве грубой оценки координат наблюдаемых целей $\left(\hat{x}_{m}, \hat{y}_{m}\right)$.

Для восьмой точки значение $\lg \left(\Delta r_{\Sigma}(\hat{x}, \hat{y})\right)$ превысило величину порога, и, следовательно, эта точка была определена как ложная цель.

Степень отклонения $\Delta \hat{x}_{m}, \Delta \hat{y}_{m}$ полученных грубых оценок $\left(\hat{x}_{m}, \hat{y}_{m}\right)$ от истинных координат цели $\left(x_{m}, y_{m}, z_{m}\right)$ представлена на рис. $6 a, 6 b$ серыми диаграммами, где по горизонтальной оси отложен номер цели. Видно, что по координате $x$ ошибка не превышает половины шага виртуального обзора по соответствующей координате, по оси $y$ ошибка значительно больше и достигает 250 м.

На рис. $6 c$ ошибка грубой оценки по координате $z$ представлена как модуль разности между истинной высотой цели $z_{m}$ и серединой зоны действия МПРЛС по высоте $\bar{z}$ и достигает значений 900 м.

Эффективность межпозиционного отождествления измерений и правильность формирования векторов измерений $\mathbf{R}_{m}$ была оценена по точности определения координат целей $\left(x_{m}^{*}, y_{m}^{*}, z_{m}^{*}\right)$ итерационным суммарно-дальномерным методом наименьших квадратов [10], для которого сформированные векторы $\mathbf{R}_{m}$ были использованы в качестве измерений для каждой $m$-й цели, а грубые оценки координат целей $\left(\hat{x}_{m}, \hat{y}_{m}, \bar{z}\right)$ применены в качестве начального решения координатной задачи.

Степень отклонения $\Delta x_{m}^{*}, \Delta y_{m}^{*}, \Delta z_{m}^{*}$ полученной точной оценки $\left(x_{m}^{*}, y_{m}^{*}, z_{m}^{*}\right)$ от заданных координат $\left(x_{m}, y_{m}, z_{m}\right)$ целей показана на рис. 6 в виде черных диаграмм. Видно, что ошибки точной оценки по координатам $x$ (рис. $6 a$ ) и $y$ (рис. $6 b$ ) сократились на порядок относительно $\Delta \hat{x}_{m}, \Delta \hat{y}_{m}$ и составляют единицы метров. Погрешность точной оценки по координате z (рис. $6 c$ ) сократилась, но не так значительно (примерно в 6 раз) и не превышает значения 150 м, что мо- 


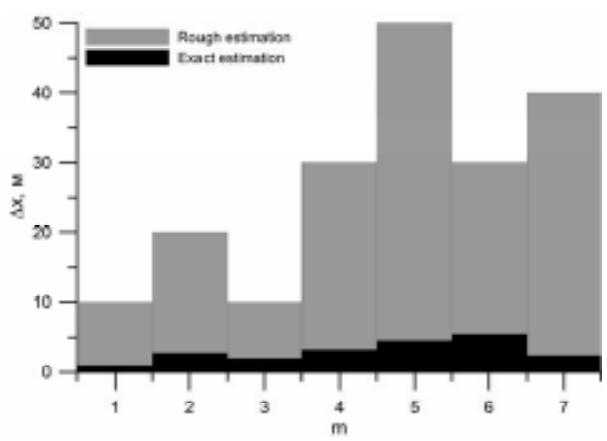

a)

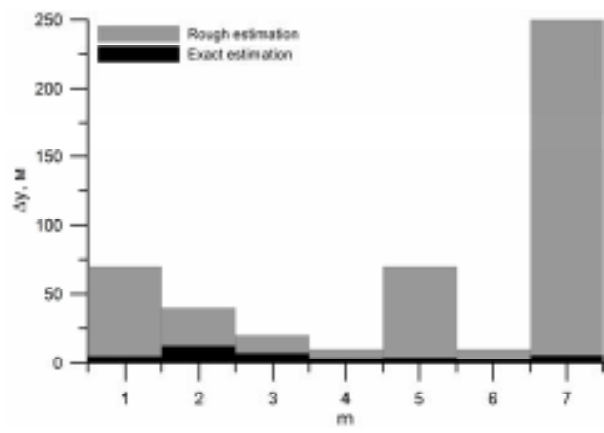

b)

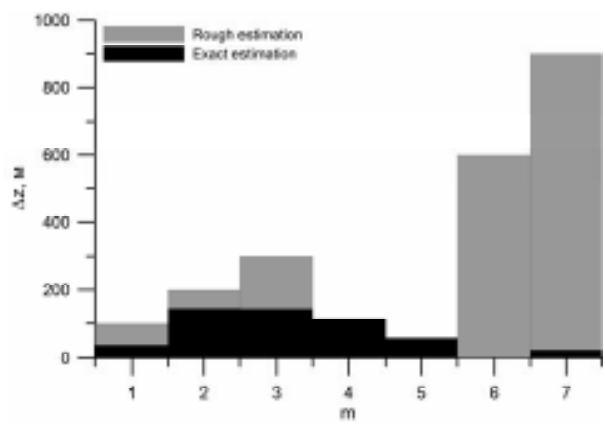

c)

Рис. 6. Степень отклонения грубых (серые диаграммы) и точных (черные диаграммы) оценок координат целей от их истинных значений: $a$ - по координате $x ; b$ - по координате $y ; c$ - по координате $z$

Fig. 6. Level of a deviation rough (gray charts) and exact (black charts) estimations of coordinates of the targets from their true values: $\mathrm{a}$ - on coordinate $x ; \mathrm{b}$ - on coordinate $y ; \mathrm{c}-$ on coordinate $z$

жет быть объяснено конфигурацией МПРЛС и погрешностью дальномерных измерений. Высокая точность полученных оценок $\left(x_{m}^{*}, y_{m}^{*}, z_{m}^{*}\right)$ координат целей свидетельствует о правильном определении принадлежности полученных в разных позициях измерений тем или иным целям и правильном формировании векторов измерений для каждой цели.

\section{Заключение}

Таким образом, в настоящей статье предложен новый способ межпозиционного отождествления измерений и предварительного определения координат воздушных целей в суммарнодальномерной МПРЛС в условиях многоцелевой обстановки, когда завязка траекторий целей еще не произошла и все цели наблюдаются одновременно. Способ основан на виртуальном обзоре пространства, использует только суммарно-дальномерные измерения и не требует измерения дополнительных параметров. Результаты, полученные в ходе численного имитационного моделирования, показывают высокую степень эффективности предложенного способа как по точности получаемой предварительной оценки координат целей, так и по правильности формирования векторов измерений для каждой наблюдаемой цели. 


\section{Список литературы}

[1] Черняк В.С. Многопозиционная радиолокаиия. М.: Радио и связь, 1993. 416 с. [Chernyak V.S. Multistation radiolocation. Moscow, Radio i sv’yaz, 1993. 416 p. (in Russian)]

[2] Просов А.В., Квиткин В.П. Алгоритмы межпозиционного отождествления результатов радиолокационных измерений. Сборник научных трудов Харьковского университета Воздушных Сил им. И. Кожедуба. Харьков, 2008, 2(17), 46-48 [Prosov A.V., Kvitkin V.P. Algorithms of an interposition identification of results of radar-tracking measurements. The collection of proceedings of the Kharkov university of Military-air forces named of I. Kozhedub, Kharkov, 2008, 2(17), 46-48 (in Russian)]

[3] Дмитренко А.А., Седышев С.Ю. Межпозиционное пространственно-временное отождествление сигналов в многопозиционных базово-корреляционных комплексах пассивной локации. Доклады БГУИР. Минск, 2016, 5(99), 85-91 [Dmitrenko A.A., Sedyshev S.Yu. Interposition existential identification of signals in multistation base-correlation complexes of a passive location. Reports BSUIR, Minsk, 2016, 5(99), 85-91 (in Russian)]

[4] Kaplan L.M., Bar-Shalom Y., Blair W.D. Assignment Costs for Multiple Sensor Track-to-Track Association. IEEE Transactions on AES. April 2008, 44(2), 655-677.

[5] Bar-Shalom Y., Blackman S., Fitzgerald R. Dimensionless Score Function for Multiple Hypothesis Tracking. IEEE Transactions on AES. Jan 2007, 43(1), 392-400.

[6] Wang H., Kirubarajan T., Bar-Shalom Y. Precision Large Scale Air Traffic Surveillance Using IMM/Assignment Estimators. IEEE Transactions on AES. Jan 1999. 35(1), 255-266.

[7] Roecker J. A. Multiple Scan Joint Probabilistic Data Association. IEEE Transactions on AES. July 1995, 31(3), 1204-1210.

[8] Меркулов В.И., Лепин В.Н. Авиационные системы радиоуправления. М.: Радио и связь, 1997. 396 c. [Merkulov V.I., Lepin V.N. Aviation systems of a radio-guidance. Moscow, Radio i sv'yaz, 1997. 396 p. (in Russian)]

[9] Кирюшкин В.В., Черепанов Д.А. Оценка координат воздушной цели в многопозиционной системе наблюдения «навигационные спутники - воздушная цель - наземный приемник». Журнал СФУ. Техника и технологии, 2016, 9(8), 1172-1182 [Kiryushkin V.V., Cherepanov D.A. Coordinates estimation of the air target in the multiitem observation system «navigation satellites the air target - the ground receiver» J. Sib. Fed. Univ. Eng. technol., 2016, 9(8), 1172-1182. DOI: 10.17516/1999-494X-2016-9-8-1172-1182 (in Russian)]

[10] Кирюшкин В.В., Супрунов А.В., Волков Н.С. Использование навигационных позиционных методов определения координат в задаче наблюдения воздушных целей. Радиолокаџия, навигация и связь: Сборник трудов ХХІV Международной научно-технической конферениии. Воронеж: Велборн, 2018, 3, 163-174 [Kiryushkin V.V., Volkov N.S., Suprunov A.V. Use of navigating position methods of definition of coordinates in the problem of observation of air targets. Radiolocation, navigation and communication: The collection of works of XXIV International scientific and technical conference. Voronezh, Velborn, 2018, 3, 163-174 (in Russian)] 